\title{
Editorial for special issue on metallurgical process engineering and intelligent manufacturing
}

\author{
An-jun $X u^{1)}$ and Yan-ping $B a o^{2)}$ \\ 1) School of Metallurgical and Ecological Engineering, University of Science and Technology Beijing, Beijing 100083, China \\ 2) State Key Laboratory of Advanced Metallurgy, University of Science and Technology Beijing, Beijing 100083, China
}

In recent years, new concepts and new technologies for improving the manufacturing level have emerged one after another, and the global industrial competition pattern will undergo major adjustments. The United States proposed the "Advanced Manufacturing Country" strategy, France proposed the "New Industry France Plan," and Germany proposed "Industry 4.0." At the same time, China's economic development has entered a new normal, and the resource and environmental constraints faced by the manufacturing industry have been continuously strengthened. In this context, the "Made in China 2025" plan was promulgated, which insists on innovation-driven, intelligent transformation, and accelerating the transition from a manufacturing power to a manufacturing power.

The metallurgical industry has entered the 21 st century, and its common proposition of the times is green and intelligent. Greening and intelligence are both integral and systematic propositions, which require the support of science and technology at the atomic/molecular level, process/device level, and especially the manufacturing process level.

Metallurgical process engineering is a new branch of metallurgical engineering, which solves complex problems in open-dynamic systems based on dissipative structure theory. The target is the overall optimization in the production process of metallurgical enterprises, including static optimization of structural framework of the manufacturing process and dynamic operation optimization of production process, as well as the organic combination of them. It solves the coordination-optimization of the set of procedures' relations and the reconstruction-optimization of the set of procedures in the manufacturing process, involving a global, overall process structure-function-efficiency optimization. Namely, it will optimize the overall strategic propositions of greenization and intelligence of metallurgical plants by intersection of physical system optimization with information engineering and environmental ecological engineering.

This special issue includes 13 papers, including 10 research papers and 3 review papers on metallurgical process engineering. All papers were mainly focused on the research development in realizing the optimization of material flow operation, energy flow operation, and information flow operation optimization in the steel manufacturing process through modeling, which provide technical support for the green and intelligent steel manufacturing process.

First of all, Academician Yin [1] summarized the three levels of the knowledge system of metallurgical science and engineering, and proposed that the development of macrodynamic metallurgy must eliminate the concept of an "isolated system" and establish the concepts of "flow," "process network," and "operating program" to study the "structurefunction-efficiency" in the macro-dynamic operation of metallurgical manufacturing processes. This means taking "flow" as the ontology and observing dynamic change by "flow" to solve the green and intelligent proposition of metallurgical enterprises. Metallurgical process engineering is a cross-level, comprehensive, and integrated study of the macro-dynamic operation of manufacturing processes. It studies the physical nature and constitutive characteristics of the dynamic operation of steel manufacturing process, as well as the analysis-optimization of the set of procedure functions, coordination-optimization of the set of procedures' relations, and reconstruction-optimization of the set of procedures in the manufacturing process. The study establishes rules for the macro-operation of the manufacturing process, as well as the dynamic and precise objectives of engineering design and production operation.

In a steel mill, the material flow is the main body processed in the manufacturing process, and the optimization and control of the material flow is an important means of reducing costs and increasing efficiency in steel mills. Lin and 
Zeng [2] elaborated on functional analysis, reasonable positioning, and process optimization of each aspect of steel. The current state of molten steel quality and implementation under narrow window control was analyzed. A method for maintaining stability in the narrow window control technology of steel quality was proposed, which was controlled by factors including composition, temperature, time, cleanliness, and consumption (raw material). This provides an important guidance for the future development of a green and intelligent steel manufacturing process. Chu and Bao [3] investigated the behaviors of both Mn evaporation and nitrogen removal from molten Mn steel via vacuum slag refining in a vacuum induction furnace. The results show that a reaction interface between denitrification and Mn evaporation tends to migrate from the surface of slag layer to the surface of molten steel with the gradual exposure of molten steel during the vacuum slag refining process. Under certain vacuum pressure, the slag shows a stronger inhibitory effect on Mn evaporation rate than that of reducing vacuum degree. Moreover, the inhibitory effect of the slag layer on Mn evaporation can be weakened with the increase of the initial Mn content. The slag layer can work as an inhibitory layer to reduce the Mn evaporation from molten steel, and the evaporation reaction of Mn mainly proceeds on the surface of the molten steel. This may be because the Mn mass transfer coefficients for one of reactions at steel-slag interface, mass transfer in molten slag, and evaporation reaction at the slag-gas interface are lower than that of evaporation reaction at the steel-gas interface. The introduction of slag is proposed for both high efficiency denitrification and improved yield of Mn during the vacuum refining process of Mn steel grades. Wang et al. [4] studied the mass transfer among the multiphase interactions for the steel, slag, lining refractory, and nonmetallic inclusions during the refining process of a bearing steel using laboratory experiments and numerical kinetic prediction. Experiments on the system with and without the slag phase were carried out to evaluate the influence of the refractory and the slag on the mass transfer. A mathematical model coupled the ion and molecule coexistence theory, coupled-reaction model, and the surface renewal theory was established to predict the dynamic mass transfer and composition transformation of the steel, the slag, and nonmetallic inclusions in the steel. The composition transformation of inclusions and the mass transfer of magnesium and aluminum in the steel were predicted with an acceptable accuracy using the established kinetic model. Wu et al. [5] studied the impact energy prediction model of low carbon steel based on industrial data. A threelayer neural network, extreme learning machine, and deep neural network were compared with different activation functions, structure parameters, and training functions. Bayesian optimization was used to determine the optimal hyper-parameters of the deep neural network. The model with the best performance was applied to investigate the import- ance of process parameter variables on the impact energy of low carbon steel. The results show that the deep neural network obtains better prediction results than the shallow neural network because the multiple hidden layers improve the learning ability of the model. Yuan et al. [6] established an improved case-based reasoning (CBR) model based on heat transfer calculation by combining the mechanism model and the data-driven model to realize the prediction of the molten steel temperature at the end of ladle furnace (LF) refining. The results show that the improved CBR model based on heat transfer calculation (CBR-HTC) improves the prediction accuracy of end-point molten steel temperature by $5.33 \%$ and $7.00 \%$ compared with the original CBR model and $6.66 \%$ and $5.33 \%$ compared with the back propagation neural network (BPNN) model in the ranges of $[-3,3]$ and $[-7,7]$, respectively.

At present, under the realistic background of overcapacity of iron and steel enterprises, imbalance of product structure, and high consumption of materials and energies, the implementation of operation optimization in the iron and steel manufacturing process is essential to reduce production costs, increase production or energy efficiency, and improve production management. To deal with the increasing demand for low-volume customization of the mechanical properties of cold-rolled products, Yan and Lü [7] proposed a process parameter design and optimization method that combines multi-objective quality prediction and process parameter optimization (PPO). This method uses the multi-output support vector regression (MSVR) method to simultaneously predict multiple quality indices. The experimental results show that, compared with the traditional single-objective quality prediction model based on support vector regression (SVR), the multi-objective prediction model could better take into account the coupling effect between process parameters and quality index, the MSVR model prediction accuracy is higher than that of the SVR, and the optimized process parameters are more capable to reflect the influence of metallurgical mechanism on the quality index, which are more in line with actual production process requirements. Lü et al. [8] proposed an available production capacity network with varietal compatibility and virtual occupancy for enhancing production plan implementation and quick adjustment in the case of dynamic production changes. The genetic algorithm improved with local search was used to optimize the proposed production plan and significantly reduce the order delay rate. The validity of the proposed model and algorithm was numerically verified by simulating actual production practices. The simulation results demonstrate that the model and improved algorithm result in an effective production plan. Yang et al. [9] established three quantitative models in response to the existing problems of the quantitative evaluation methods for multi-process collaborative operations. the multi-process collaborative operation level was evaluated through the lam- 
inar-flow operation degree, the process matching degree, and the scheduling strategy availability degree. Based on actual production data, the average laminar-flow operation (process matching) degrees of different steel plants were evaluated. On this basis, a scheduling strategy based on the optimization of the furnace-caster coordinating mode was suggested. Simulation experiments showed higher availability than the greedy-based and manual strategies. The multi-process collaborative operation level was improved with fewer adjustments and interruptions in casting. He et al. [10] put forward a whole set of intelligent scheme for bar warehouse crane for the guidance of metallurgical process engineering, including cluster rapid self-awareness technology of the smart crane, precise self-executing technique of crane with rigid-flexible hybrid structure, multi-body system kinematics model of the smart crane sling and the swing characteristics model at different azimuth, anti-swing control technology based on the optimization objective function, the vehicle model recognition system based on lidar, and the clustering crane dynamic scheduling method based on multi-agent reinforcement learning. The complete intelligent logistics system of the bar warehouse has changed the original operation mode of the warehouse area and realized the unmanned operation and intelligent scheduling of the crane, which is of great significance for improving the production efficiency, reducing the production cost, and improving the product quality. Xu et al. [11] analyzed the operation optimization problem of the steel manufacturing process, which needed to go through a complex production organization from customers' orders to workshop production. The existing research on the operation optimization techniques, including process simulation, production planning, production scheduling, interface scheduling, and scheduling of auxiliary equipment, was reviewed. The literature review reveals that, although considerable research has been conducted to optimize the operation of steel production, these techniques are usually independent and unsystematic. Therefore, the future work related to operation optimization of the steel manufacturing process based on the integration of multi technologies and the intersection of multi disciplines were summarized.

The production process of iron and steel is accompanied by a large amount of energy production and consumption. Optimal scheduling and utilization of these energies within energy systems are crucial to realize a reduction in the cost, energy use, and $\mathrm{CO}_{2}$ emissions. Liu et al. [12] proposed an efficient multi-layer model based on the energy hub (EH) concept, which is designed to systematically model the energy system and schedule energy within steelworks to meet the energy demand. Besides, to simulate the actual working conditions of the energy devices, the method of fitting the curve is used to describe the efficiency of the energy devices. Moreover, to evaluate the applicability of the proposed model, a case study is conducted to minimize both the economic operation cost and $\mathrm{CO}_{2}$ emissions. The optimal results demonstrated that the model is suitable for energy systems within steel works. Further, the economic operation cost decreased by $3.41 \%$, and $\mathrm{CO}_{2}$ emissions decreased by approximately $3.67 \%$.

Information flow is the sum of material behavior, energy behavior, reflection of external environmental information, and man-made control information. Steel production involves the transfer and transformation of material and energy at different levels, structures, and scales, and also involves the interaction between material and energy. This process incurs substantial information in the material and energy dimensions. Given the black-box feature of iron and steel production processes, process visualization plays an important role in the continuous development of virtual reality (VR) technology and will inevitably benefit parameter correction, technical support decision-making, personnel training, and other aspects of the steel metallurgy industry. Xu et al. [13] analyzed the technological characteristics of the entire process in the iron and steel metallurgical industry, whose final products are coils or sheets. First, a visualization technology route based on VR was built. On the basis of the characteristics of VR technology, a visual simulation model for the process scheduling of the iron and steel enterprise raw materials' field, slab, and hot rolling processes was built. Next, a visualization simulation platform of the iron and steel metallurgy plant-wide process, including ironmaking, steelmaking, hot rolling, and cold rolling, was developed. Lastly, a visualization simulation platform for future application and development prospects was presented.

This special issue introduces some of the latest developments in metallurgical process engineering, which are believed to provide theoretical and technical support for the green and intelligent steel manufacturing process. We sincerely thank all the authors for their innovative works and all the reviewers for their criticisms and comments. We would like to thank the Editorial Team of IJMMM for their hard work to get this special issue published.

\section{References}

[1] R.Y. Yin, Review on the study of metallurgical process engineering, Int. J. Miner. Metall. Mater., 28(2021), No. 8, p. 1253.

[2] L. Lin and J.Q. Zeng, Consideration of green intelligent steel processes and narrow window stability control technology on steel quality, Int. J. Miner. Metall. Mater., 28(2021), No. 8, p. 1264.

[3] J.H. Chu and Y.P. Bao, Mn evaporation and denitrification behaviors of molten $\mathrm{Mn}$ steel in the vacuum refining with slag, Int. J. Miner. Metall. Mater., 28(2021), No. 8, p. 1288.

[4] J.J. Wang, L.F. Zhang, G. Cheng, Q. Ren, and Y. Ren, Dynamic mass variation and multiphase interaction among steel, slag, lining refractory and nonmetallic inclusions: Laboratory experiments and mathematical prediction, Int. J. Miner. Metall. Mater., 28(2021), No. 8, p. 1298. 
[5] S.W. Wu, J. Yang, and G.M. Cao, Prediction of the Charpy Vnotch impact energy of low carbon steel using a shallow neural network and deep learning, Int. J. Miner. Metall. Mater., 28(2021), No. 8, p. 1309.

[6] F. Yuan, A.J. Xu, and M.Q. Gu, Development of an improved CBR model for predicting steel temperature in ladle furnace refining, Int. J. Miner. Metall. Mater., 28(2021), No. 8, p. 1321.

[7] Y.F. Yan and Z.M. Lü, Multi-objective quality control method for cold-rolled products oriented to customized requirements, Int. J. Miner. Metall. Mater., 28(2021), No. 8, p. 1332.

[8] Z.M. Lü, T.R. Jiang, and Z.W. Li, Multiproduct and multistage integrated production planning model and algorithm based on an available production capacity network, Int. J. Miner. Metall. Mater., 28(2021), No. 8, p. 1343.

[9] J.P. Yang, Q. Liu, W.D. Guo, and J.G. Zhang, Quantitative evaluation of multi-process collaborative operation in steelmaking-continuous casting sections, Int. J. Miner. Metall. Mater., 28(2021), No. 8, p. 1353.

[10] H.N. He, X.C. Wang, G.Z. Peng, D. Xu, Y. Liu, M. Jiang, Z.D. $\mathrm{Wu}, \mathrm{D}$. Zhang, and $\mathrm{H}$. Yan, Intelligent logistics system of steel bar warehouse based on ubiquitous information, Int. J. Miner. Metall. Mater., 28(2021), No. 8, p. 1367.

[11] Z.J. Xu, Z. Zheng, and X.Q. Gao, Operation optimization of the steel manufacturing process: A brief review, Int. J. Miner. Metall. Mater., 28(2021), No. 8, p. 1274.

[12] S. Liu, S. Xie, and Q. Zhang, Multi-energy synergistic optimization in steelmaking process based on energy hub concept, Int.
J. Miner. Metall. Mater., 28(2021), No. 8, p. 1378.

[13] T. Xu, G. Song, Y. Yang, P.X. Ge, and L.X. Tang, Visualization and simulation of steel metallurgy processes, Int. J. Miner. Metall. Mater., 28(2021), No. 8, p. 1387.

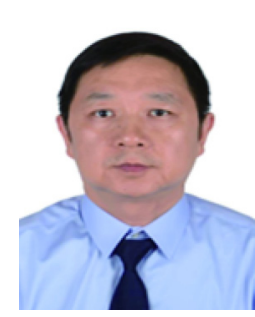

An-jun $\mathbf{X u}$ is a professor at University of Science and Technology Beijing, China since 2013. He is the leader of metallurgical process engineering research team and has published over 200 papers. His main research interests and contributions are steelmaking technology and process optimization. He is one of the presidents of metallurgical process engineering committee and secretary of steelmaking committee of the Chinese Society for Metals (CSM).

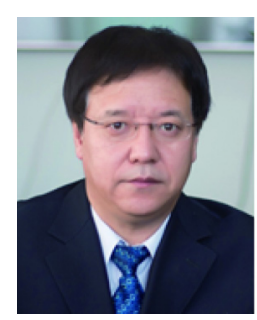

Yan-ping Bao is a professor at University of Science and Technology Beijing, China since 2001. He has published over 200 papers. His main research interests and contributions are steelmaking process optimization, alloy reduction applications, and steel cleanliness control. He is the first Chinese professor to win the "Willy Korf Excellent lecture Aaward" in 2012. 